\title{
On Exact Kerr-Schild Type Vacuum Solutions to Salam's Two-Tensor Theory of Gravity
}

\author{
R. Mansouri and H. K. UrbantKe \\ Institut für Theoretische Physik der Universität, Wien
}

Received January 17, 1972

Abstract. A Kerr-Schild type ansatz for the $f$ and $g$ tensor fields leads to a tractable form of the field equations of Salam's two-tensor theory of gravity in vacuo. While the general solution contains the Schwarzschild and Kerr metrics in the pure Einstein vacuum case, we can show in the $f-g$ case that all "non-trivial" $(f \neq g)$ solutions are restricted to have the form of "plane-fronted waves".

\section{Introduction and Conclusion}

A two-tensor theory has been set up by Salam et al. [1] to describe the gravitational interactions of leptons and hadrons. Its field equations in regions free from matter are

where

$$
\begin{aligned}
& G_{\mu v}(f)+\kappa_{f}^{2}\left|\operatorname{det} f_{\mu \nu}\right|^{-\frac{1}{2}} \frac{\partial \mathscr{L}_{f g}}{\partial f^{\mu \nu}}=0, \\
& G_{\mu v}(g)+\kappa_{g}^{2}\left|\operatorname{det} g_{\mu \nu}\right|^{-\frac{1}{2}} \frac{\partial \mathscr{L}_{f g}}{\partial g^{\mu \nu}}=0 .
\end{aligned}
$$

$$
\begin{aligned}
\mathscr{L}_{f g} & \equiv \kappa_{f}^{-2}\left|\operatorname{det} f_{\mu \nu}\right|^{\frac{1}{2}} \frac{m^{2}}{4}\left(f^{\alpha \beta}-g^{\alpha \beta}\right)\left(f^{\kappa \lambda}-g^{\kappa \lambda}\right)\left(g_{\alpha \kappa} g_{\beta \lambda}-g_{\alpha \beta} g_{\kappa \lambda}\right), \\
\frac{\partial \mathscr{L}_{f g}}{\partial f^{\mu \nu}} & =\left(-\frac{1}{2} \delta_{\mu}^{\alpha} \mathscr{L}_{f g}+\frac{m^{2}}{2 \kappa_{f}} \mathscr{F}_{\mu}^{\alpha}\right) f_{\alpha \nu} \\
\frac{\partial \mathscr{L}_{f g}}{\partial g^{\mu \nu}} & =-\frac{m^{2}}{2 \kappa_{f}} \mathscr{F}_{\mu}^{\alpha} g_{\alpha \nu}, \\
\mathscr{F}_{\mu}^{\alpha} & \equiv \kappa_{f}^{-1}\left|\operatorname{det} f_{\mu \nu}\right|^{\frac{1}{2}}\left[g_{\mu \varrho} f^{\varrho \sigma} g_{\sigma \tau} f^{\tau \alpha}-g_{\mu \varrho} f^{\varrho \alpha}\left(g_{\kappa \lambda} f^{\lambda \kappa}-3\right)\right],
\end{aligned}
$$

$G_{\mu \nu}(f), G_{\mu \nu}(g)$ are the Einstein tensors constructed from the symmetric nonsingular tensor fields $f_{\mu \nu}, g_{\mu \nu}$ when considered as "metrics". $m>0$ is the mass of the $f$-field which in regions containing matter would interact directly only with hadrons, while $g$ would interact directly only with

\footnotetext{
* Supported by „Fonds zur Förd. d. wiss. Forsch. in Österr.”. Nr. 1255.
} 
leptons. Thus the gravitational interaction between leptons and hadrons goes via the "mixing terms" on the right hand side of Eqs. (1.1).

Several attempts have been reported $[2,3]$ to find exact nontrivial solutions (i.e. $f_{\mu \nu} \neq g_{\mu \nu}$ ) of the vacuum equations (1.1), which are rather complicated. One typical approach is to impose symmetry conditions to simplify (1.1), but so far there was not too much success. Another approach consists in imposing algebraic assumptions on $f, g$. As an example of this approach, we consider the case where $f$ and $g$ are of the Kerr-Schild $[4,5]$ form, i.e.

$$
\begin{aligned}
& g=\eta+2 \mathscr{G} k \otimes k, \\
& f=\eta+2 \mathscr{F} k \otimes k,
\end{aligned}
$$

where $\eta$ is the flat Minkowski metric and $k$ is a field of Minkowski null vectors $\left(\eta^{\mu \nu} k_{\mu} k_{v}=0\right)$. The reason for doing so is that on the one hand the class of Kerr-Schild metrics in general relativity contains several interesting metrics such as the plane-fronted waves $[6,7]$, the Vaidya metric $[8,9]$ and the Kerr family $[10,5]$, and can be explicitly determined in the Einstein vacuum case $[4,5]$. On the other hand, the drastic simplification of $(1.1,2)$ when $(1.3)$ is inserted allows a complete treatment in the $f-g$ case.

The result, however, is rather poor when compared with the corresponding pure Einstein vacuum case. Namely, Eqs. (1.1) permit only Kerr-Schild solutions which are plane-fronted [7] waves, i.e. fields containing a geodesic null congruence with vanishing shear, twist and expansion and with a Weyl tensor of the algebraic type $N[11,12]$. Two cases are to be distinguished. The first is characterized by vanishing rotation $[7,12]$ of the congruence, i.e. parallel rays (" $p p$ waves"), and has been described earlier [3]. In the second case the rotation does not vanish. Choosing the coordinates in Kundt's [7] canonical way, the solution can be written

where

$$
\begin{aligned}
& g_{\mu \nu} d x^{\mu} d x^{\nu}=d s_{0}^{2}+x \mathscr{G} d u^{2}, \\
& f_{\mu \nu} d x^{\mu} d x^{\nu}=d s_{0}^{2}+x \mathscr{F} d u^{2},
\end{aligned}
$$

$$
d s_{0}^{2}=2 d u d v+\left|d z-2 v x^{-1} d u\right|^{2}-3 v^{2} x^{-2} d u^{2}
$$

is the flat metric in some noninertial coordinates $(z \equiv x+i y), \mathscr{G}=\mathscr{G}(u, x, y)$ and $\mathscr{F}(u, x, y)$ are arbitrary functions of $u$ whose $x, y$-dependence is given by $\left(\Delta_{2}=\partial^{2} / \partial x^{2}+\partial^{2} / \partial y^{2}\right)$

$$
\begin{aligned}
& \Delta_{2} \mathscr{F}=m^{2}(\mathscr{F}-\mathscr{G}), \\
& \Delta_{2} \mathscr{G}=-\left(\kappa_{g}^{2} / \kappa_{f}^{2}\right) m^{2}(\mathscr{F}-\mathscr{G}) .
\end{aligned}
$$

Equation (1.6) is formally the same as in the $p p$ case [3] and can easily be decoupled and solved for suitable boundary conditions. 
In the rest of the paper we want to prove our statements made above. Several obvious generalizations suggest themselves which will be treated elsewhere; it is, for instance, easy to read off type III $f-g$ solutions from Ref. [7]. The interest in all the solutions obtainable in this way lies not so much in the particular solutions that might result, but in the clarification of the general structural properties of the underlying theory [although some solutions which appear as a byproduct might be interesting in themselves; see the Kerr example]. One might, e.g., think that not only our very special kind of ansatz (1.3), but also the particular form of the mixing terms (1.2) (stemming from "covariantization" of $f_{\mu \nu}-\eta_{\mu \nu}$ ) might be a reason why the class considered here is not too rich. It seems worth while to test this by considering the various generalizations.

Finally we want to mention that all what has been said about solutions of (1.1) of the form (1.3) remains valid in the two-tensor theory of massive gravitation [13], a covariant extension of the theory of Freund et al. [14].

Acknowledgment. We wish to thank Dr. P. Aichelburg for many helpful discussions and for drawing our attention to Ref. [5].

\section{Determination of the Optical Scalars and the Petrov Type}

Assume that $g_{\mu v}, f_{\mu v}$ are of the form (1.3); then

$$
f_{\mu \nu}-g_{\mu \nu}=2 \mathscr{H} k_{\mu} k_{v} \quad \text { with } \quad \mathscr{H} \equiv \mathscr{F}-\mathscr{G} .
$$

The field equations (1.1) now simplify drastically ${ }^{1}$, reducing to

$$
G_{\mu \nu}(f)=m^{2} \mathscr{H} k_{\mu} k_{v}, \quad G_{\mu \nu}(g)=-\frac{\kappa_{g}^{2}}{\kappa_{f}^{2}} m^{2} \mathscr{H} k_{\mu} k_{v} .
$$

Obviously, both $\mathscr{F}$ and $\mathscr{G}$ must not vanish for nontrivial solutions $(\mathscr{H} \neq 0)$. By contraction, both curvature scalars vanish, and (2.2) is equivalent to

$$
R_{\mu v}(f)=m^{2} \mathscr{H} k_{\mu} k_{v}, \quad R_{\mu \nu}(g)=-\frac{\kappa_{g}^{2}}{\kappa_{f}^{2}} m^{2} \mathscr{H} k_{\mu} k_{v} .
$$

The contracted Bianchi identities imply from (2.2) that $k_{\mu}$ is geodesic.

For further analysis, we use the tetrad formalism of Sec. 5 of Ref. [5] (henceforth referred to as $K S$ ), the function $h$ appearing there replaced

1 This simplification is purely algebraic, depending only on a relation like (2.1) with $k$ a null vector of $g$, but not on (1.3). Here is a starting point for generalizations. 
by $\mathscr{G}$ or $\mathscr{F}$ for $g_{\mu v}, f_{\mu v}$. The two tetrads adapted to $g, f$ differ only in the vector $\partial_{3}=\partial_{u}-h \partial_{4}$; note, however, that the effect of both $\partial_{3}$ 's on functions $F$ with $\partial_{4} F=0$ is the same (this remark will remove most of the apparent ambiguities that might arise in the following, as we will not distinguish the tetrads and indices for notational convenience). The tetrad form of $(2.3)$ is then

$$
\begin{aligned}
& R_{a b}(f)=0=R_{a b}(g) \quad \text { for } \quad(a, b) \neq(3,3), \\
& R_{33}(f)=m^{2} \mathscr{H}, \\
& R_{33}(g)=-\frac{\kappa_{g}^{2}}{\kappa_{f}^{2}} m^{2} \mathscr{H} \text {. }
\end{aligned}
$$

When we set $(K S(5.7))$

$$
k_{\mu} d x^{\mu}=e^{3}=d u+\bar{Y} d \xi+Y d \bar{\xi}-Y \bar{Y} d v,
$$

the geodesic condition mentioned aboved reads $Y_{, 4}=-\Gamma_{424}=0$ (and complex conjugate). With this, $k_{\mu}$ given by (2.4) is automatically affinely parametrized, i.e. $k_{\mu ; v} k^{v}=0$. The contracted Bianchi identity applied to (2.2) then leaves

$$
\mathscr{H}_{, 4}+\mathscr{H}^{v}{ }_{; v}=0 .
$$

(Here $\mathscr{H}_{, 4}=k^{\mu} \partial_{\mu} \mathscr{H}$; again there is no ambiguity; all optical scalars of $k_{\mu}$ are the same with respect to both metrics!)

Now we show that the shear of $k_{\mu}$ must vanish for nontrivial solutions. Indeed, the complex shear in this tetrad formalism is given by $Y_{, 2}$, and the field equations $R_{22}=0$ imply

$$
Y_{, 2}\left[\mathscr{F}_{, 4}+(\bar{Z}-Z) \mathscr{F}\right]=Y_{, 2}\left[\mathscr{G}_{, 4}+(\bar{Z}-Z) \mathscr{G}\right]=0
$$

(cf. $K S(5.25)$ ). Assuming $Y_{, 2} \neq 0$, we would have

$$
\pi_{, 4}=0=\mathscr{G}_{, 4} \quad Z=\bar{Z},
$$

so that the imaginary part of $Z$ would vanish, but according to (2.5) also its real part, the divergence or expansion. Since now $Z=Y_{, 1}=0$, the integrability condition $K S(2.7)$ applied to $Y_{,[14]}$ gives $Y_{, 2} \bar{Y}_{, 1}=\left|Y_{, 2}\right|^{2}=0$, a contradiction. Therefore we must have vanishing shear: $Y_{, 2}=0$. Now the equations $R_{44}=R_{42}=R_{22}=0$ (and c.c.) are satisfied.

Next we show that the complex expansion $Z$ must vanish also. Our procedure is to assume $Z \neq 0$, which enables us to follow $K S(5)$ further: we satisfy the equations $R_{12}=R_{34}=0$ by setting $h=\frac{1}{2} M(Z+\bar{Z})$ with $M_{, 4}=0$, i.e.

with

$$
\mathscr{F}=\frac{1}{2} L(Z+\bar{Z}), \quad \mathscr{G}=\frac{1}{2} N(Z+\bar{Z})
$$

$$
L_{, 4}=N_{, 4}=0 \text {. }
$$


If now $\mathscr{H}=\mathscr{F}-\mathscr{G}$ and $\mathscr{H}, 4$ are formed and, together with $Z+\bar{Z}=k^{\mu}{ }_{; \mu}$ and $Z_{, 4}=-Z^{2}(K S(5.34))$, inserted into (2.5), we obtain

$$
(L-N) Z \bar{Z}=0 \text {. }
$$

Thus, the assumption $Z \neq 0$ leads to trivial solutions, $L=N$, proving our assertion.

It is now seen from the expressions for the relevant components of the Riemann or Weyl tensor that the field equations together with $Z=0$ imply Petrov type $N$ with $k_{\mu}$ as a quadruple Debever-Penrose vector.

\section{Canonical Form of Solutions}

Kundt [7] has determined all metrics which contain a geodesic null congruence of vanishing shear, twist and expansion and whose Ricci tensor is of the form

$$
R_{i v}=-\mu k_{i} k_{v},
$$

where $k_{\mu}$ is tangent to the null congruence. In particular, he has given a geometric definition of the concept of plane-fronted waves, and has shown that the plane-fronted waves are identical with that subclass of his metrics where the Weyl tensor has type $N$. Two essentially different cases have to be distinguished, for each of which he has given a canonical form of the metric, whereby the task of solving Einstein's equations is reduced to solving Laplace-Poisson-type equations. Our results of Sec. II show that we are dealing with $g_{\mu \nu}, f_{\mu \nu}$ which have exactly these characteristics of planefronted waves. What remains to be done is to write down our fields in Kundt's canonical form and to solve those field equations which are not yet identically satisfied.

The two essentially different cases are distinguished by the vanishing or nonvanishing of an additional optical scalar which appears when both, complex shear and expansion, vanish: the rotation $|\Omega|$ (this name is often used for the imaginary part of $Z$ also, notably by $K S$, but we should like to use the word "twist" for the latter; $|\Omega|$ is equal to $\left|Y_{, 3}\right|$ in the $K S$ formalism). The case of vanishing rotation, i.e. planefronted waves with parallel rays (" $p p$ waves"), appears in the Kerr-Schild form in Kundt's canonical coordinates; its generalization to $f-g$ solutions has been described earlier [3].

In the case of non-vanishing rotation, this quantity can be used as invariant coordinate, and the corresponding canonical form of the metric is

where

$$
\begin{gathered}
d s^{2}=\eta+A d u^{2}, \\
\eta=\left|d z-2 v x^{-1} d u\right|^{2}+2 d v d u-3 v^{2} x^{-2} d u^{2}
\end{gathered}
$$


is the flat metric in some curvilinear coordinates $(z \equiv x+i y)$, as one easily checks by calculating the Rieman tensor; it is even easy to find a transformation to Minkowski coordinates and discuss its meaning. The null congruence is given by $k_{\mu} d x^{\mu} \times d u$. The function $A$ is independent of $v$, depends arbitrarily on $u$, and to satisfy (3.1) one must have

$$
x\left(\frac{\partial^{2}}{\partial x^{2}}+\frac{\partial^{2}}{\partial y^{2}}\right)\left(x^{-1} A\right)=2 \mu .
$$

It is almost obvious now that the corresponding $f-g$ solution is given by $(1.4,5,6)$. Actually, one has to check that $f, g$ can be brought to the canonical form $(3.2,3)$ simultaneously, but this follows from the tensor relation (2.1). The function $A$ belonging to $g$, say, is independent of $v$ in the canonical coordinates. The transformation achieving this leaves open a possible $v$ dependence in the corresponding function for $f$, and (3.1) is still satisfied. But as for our field equations (2.3) $\mu \propto \mathscr{F}-\mathscr{G}$, this $v$ dependence must actually be absent. Therefore $(1.4,5,6)$ is the general solution.

\section{References}

1. Isham, C.J., Salam, A., Strathdee, J.: Phys. Rev. D3, 867 (1971).

2. Pirani,F.A.E.: Contribution to the Topical Meeting on Gravitation and Field Theory, Trieste 1971.

3. Aichelburg,P.C., Mansouri, R., Urbantke,H. K.: Phys. Rev. Letters 27, 1533 (1971).

4. Kerr, R.P., Schild, A.: Proceedings of the Meeting on General Relativity (Anniversary Volume, Fourth Centenary of Galileo's Birth) G. Barbèra, Ed. (Firenze 1965).

5. Debney, G.C., Kerr, R.P., Schild, A.: J. Math. Phys. 10, 1842 (1969).

6. Jordan,P., Ehlers. J., Kundt, W.: Akad. Wiss. Mainz, Abh. Math.-Nat. Kl. No. 2, (1960).

7. Kundt, W.: Z. Physik 163, 77 (1961).

8. Lindquist, R.W., Schwartz, R.A., Misner,C.W.: Phys. Rev. 137B, 1364 (1965).

9. Misra, M.: Proc. Roy. Irish Acad. 69, A3, 39 (1970).

10. Carter, B.: Phys. Rev. 174, 1559 (1968).

11. Pirani,F.A.E.: Brandeis Lectures 1964, Vol. I; S.Deser, K.W.Ford, Ed. New Jersey: Prentice-Hall, 1965.

12. Jordan, P., Ehlers,J., Sachs, R.K.: Akad. Wiss. Mainz, Abh. Math.-Nat. Kl. No. 1, (1961).

13. Aichelburg,P.C., Mansouri, R.: Proceedings of the Topical Meeting on Gravitation and Field Theory, Trieste (1971) (IC/71/144).

14. Freund,P. G. O., Maheshwari, A., Schonberg, E.: Astrophys. J. 157, 857 (1969).

H. K. Urbantke

Institut für Theoretische Physik

der Universität

A-1090 Wien

Boltzmanngasse 5

Austria 